\title{
Signal tranducers and activators of transcription: Expression and function in anti-neutrophil cytoplasmic antibody-associated vasculitis
}

\author{
BENJAMIN WILDE ${ }^{1}$, ANDRÉ HOERNING ${ }^{2}$, ANDREAS KRIBBEN $^{1}$, OLIVER WITZKE $^{1}$ and SEBASTIAN DOLFF \\ ${ }^{1}$ Department of Nephrology, University Hospital Essen, University of Duisburg-Essen; ${ }^{2}$ Department of Pediatrics II, \\ Pediatric Nephrology, Gastroenterology, Endocrinology and Transplant Medicine, Children's Hospital Essen, \\ University of Duisburg-Essen, Essen, North Rhine-Westphalia 45122, Germany
}

Received September 21, 2013; Accepted February 7, 2014

DOI: $10.3892 / \mathrm{mmr} .2014 .2062$

\begin{abstract}
Anti-neutrophil cytoplasmic antibody-associated vasculitis (AAV) is an autoimmune small vessel vasculitis. Despite the role of autoantibodies, T cells play an essential role in pathogenesis and are abnormal in AAV. Signal transducers and activators of transcription (STAT) affect immunoregulation and T-cell homeostasis. Therefore, the present study examined the ex vivo basal expression of the activated forms of STAT5 [phosphorylated (p)STAT5] and STAT3 (pSTAT3) in AAV and analyzed the function of two signaling pathways linked to these transcription factors. In total, 31 patients with AAV and 16 age-matched healthy controls (HCs) were enrolled. $\mathrm{CD}^{+} \mathrm{T}$ cells from peripheral blood were analyzed directly ex vivo by a fluorescence-activated cell sorter for basal expression of pSTAT5 and pSTAT3. Expression was also analyzed in $\mathrm{T}$ cells following short-term stimulation with interleukin (IL)-2 or -10. The basal expression of pSTAT5/3 in T cells was similar to AAV patients and HC. Following stimulation with IL-2 or -10 , expression of pSTAT5/3 increased in AAV subjects compared with HC. Basal expression of pSTAT3 correlated with the relapse rate in AAV. In conclusion, STAT3 and STAT5 mediated signaling pathways were functionally intact in AAV patients and exhibited hyper-responsiveness to IL-2 and -10 stimuli. Thus, T-cell abnormalities in AAV are not promoted by an altered basal expression of pSTAT $5 / 3$ or dysfunction of the IL-2/-10 signaling pathways, in which STAT $5 / 3$ are essential.
\end{abstract}

Correspondence to: Dr Benjamin Wilde, Department of Nephrology, University HospitalEssen, University of Duisburg-Essen, Hufelandstraße 55, Essen, North Rhine-Westphalia 45122, Germany E-mail: benjamin.wilde@uk-essen.de

Key words: anti-neutrophil cytoplasmic antibody, vasculitis, phosphorylated signal tranducer and activator of transcription 5, phosphorylated signal tranducer and activator of transcription 3

\section{Introduction}

Anti-neutrophil cytoplasmic antibody (ANCA)-associated vasculitis (AAV) is a necrotizing small vessel vasculitis of autoimmune origin, characterized by the presence of anti-neutrophil cytoplasmic antibodies (1). ANCAs play a pivotal pathogenic role in AAV and typically exist as two types: ANCAs with specificity for proteinase-3 (PR3) and ANCAs with specificity for myeloperoxidase (MPO) (1). Despite the importance of autoantibodies in $\mathrm{AAV}$, a previous study has indicated that $\mathrm{T}$ cells may also act as pathogenic factors in AAV (2). The isotype of ANCAs suggests that a T-cell-dependent class switch may take place (3). T cells are found in AAV organ lesions and granuloma formation, regarded as a T-cell-dependent process, is a key feature of AAV disease subtypes (2,4-6). Accordingly, T cells from AAV patients exhibit abnormal phenotype and abnormal polarization (2,7-9). T-helper (Th) cells are chronically activated in $\mathrm{AAV}$, indicated by an increased number of pro-inflammatory effector memory Th cells and upregulation of activation markers $(2,10-12)$. By contrast, anti-inflammatory $\mathrm{T}$ cells, i.e. regulatory $\mathrm{T}$ cells (Treg cells), appear to be impaired in function $(13,14)$.

Signal transducers and activators of transcription (STATs) are molecules involved in cytokine signaling cascades $(15,16)$, and are activated by phosphorylation in the cytoplasm by Janus kinases (JAKs) $(15,16)$. JAKs associate with type I and II cytokine receptors on the cytoplasmic tail, and phosphorylate STATs upon cytokine binding. STATs translocate to the nucleus and enhance or suppress transcription-specific genes $(15,16)$. Therefore, STATs are important regulators of the immune system. Interleukin (IL)-2 signaling is transduced via STAT5, and phosphorylated (p)STAT5 enhances forkhead box P3 (FOXP3) transcription, promoting the development of anti-inflammatory Treg cells $(15,17,18)$. pSTAT3 is closely linked to the development of pro-inflammatory $\mathrm{T}_{\mathrm{h}} 17$ cells and is suggested to enhance the transcription of the IL-17A/F heterodimer (18). In human inflammatory bowel disease, increased pSTAT3 levels of lesional T cells are considered to be pathophysiologically relevant (19). In addition, the balance between STAT5 and STAT3 is crucial for lineage commitment 
of T cells (18). However, IL-10 signaling also involves STAT3, which directly or indirectly leads to diminished transcription of tumor necrosis factor- $\alpha$ and decreased T-cell activation and proliferation $(20,21)$.

As STATs regulate T-cell immunity and affect immune tolerance, we hypothesized that aberrant constitutive expression of pSTAT5/3 may contribute to Th 17 expansion and to a relative deficit of Treg cells in AAV. In addition, we hypothesized that an impaired pSTAT5 response to IL-2 stimulation may contribute to the observed Treg cell dysfunction in AAV and that a decreased $\mathrm{T}$-cell response to the anti inflammatory cytokine, IL-10, (as measured by the pSTAT3 response) may promote the persistent T-cell activation frequently observed in AAV. Therefore, the present study analyzed the constitutive and induced expression of pSTAT5/3 in T cells of AAV patients.

\section{Materials and methods}

Patient cohort. A total of 31 consecutive patients with AAV visiting the outpatient clinic of the Department of Nephrology (University Hospital Essen, Essen, Germany) were enrolled in the study (mean age, 59 \pm 14 years; 20 males and 11 females). All patients were in remission at the time of sampling. In total, 26 patients had PR3-ANCA and 4 patients had ANCA with specificity for MPO at the time of diagnosis and one patient was ANCA negative. All patients were administered maintenance therapy and treated with methoxtrexate, mycophenolate, low dose cyclophosphamide or azathioprin. The patients also received low dose steroids ( $<10 \mathrm{mg} /$ day). A total of 6 patients suffered from localized disease and non-renal AAV, while the remaining 25 patients exhibited systemic AAV with renal involvement, based on the definitions of Hellmich et al (22). A diagnosis of AAV was made according to the criteria of the American College of Rheumatology and Chapel Hill Consensus (23-25). Clinical data were obtained from patient file records. In addition, 16 age-matched healthy individuals (mean age, $51 \pm 13$ years; 6 males and 10 females) with no history of chronic infection, cancer or autoimmune disease were used as the control cohort. Informed consent and approval by the local ethics committee were obtained (University Hospital Essen).

Flow cytometry: Phosflow staining for pSTAT. Expression levels of pSTAT were measured by multi-color surface staining of unstimulated and stimulated peripheral blood mononuclear cells (PBMCs). Briefly, PBMCs were isolated by density gradient centrifugation. Cells were cultured for $10 \mathrm{~min}$ in RPMI-1640 medium (Gibco-BRL, Carlsbad, CA, USA) supplemented with $5 \%$ fetal calf serum (Biowest, Renningen, Germany) in the presence of IL-10 (100 ng/ml; R\&D Systems, Wiesbaden, Germany) or IL-2 (100 ng/ml; R\&D Systems) or without any stimuli, at $37^{\circ} \mathrm{C}$ and $5 \% \mathrm{CO}_{2}$.

Phycoerythrin (PE), peridin chlorophyll protein (PerCP) and Alexa Fluor 647-labeled antibodies with various specificities were used (BD Biosciences, Heidelberg, Germany): Cluster of differentiation 4 (CD4; mouse IgG1 and PerCP), CD3 (mouse IgG1 and PerCP), pSTAT5 (Y694; clone 47; mouse IgG1 and PE) and pSTAT3 (Y704; clone 4/P-STAT3; mouse IgG1 and Alex Fluor 647).
The staining was performed according to the Phosflow III instructions (BD Biosciences) (26). Briefly, PBMCs were fixed immediately following short-term culture with pre-warmed Cytofix buffer (BD Biosciences) and subsequently permeabilized with Phosflow Perm buffer III (BD Biosciences). Next, cells were washed and stained with antibodies, followed by an incubation period of $30 \mathrm{~min}$ in the dark. Measurements were performed with a fluorescence-activated cell sorter (FACS) Calibur $^{\mathrm{TM}}$ (BD Biosciences). FACS data were analyzed by FlowJo version 7.6.5 software (Treestar Inc., Ashland, OR, USA). Expression levels are given as the mean fluorescence intensity (MFI). The response to cytokine stimulation was calculated as follows and given as a ratio: pSTAT5 MFI of IL-2-stimulated $\mathrm{T}$ cells divided by the pSTAT5 MFI of unstimulated T cells, or pSTAT3 MFI of IL-10-stimulated T cells divided by the pSTAT3 MFI of unstimulated T cells.

Statistical analysis. All values are expressed as the mean \pm standard deviation. Statistical significance of the differences between groups was determined using the Mann-Whitney U test. Spearman's rank correlation coefficient was calculated to measure the correlation between various study parameters.

\section{Results}

Constitutive levels of phosphorylated STAT5 in T cells are comparable between AAV patients and healthy controls $(H C s)$. The ex vivo basal levels of pSTAT5 were directly assessed by flow cytometry. There was no statistically significant difference between AAV patients and HCs with regard to constitutive pSTAT5 levels of $\mathrm{CD}^{+} \mathrm{T}$ cells (given as MFI; $191 \pm 143$ vs. $153 \pm 100$; $\mathrm{P}=0.5$; Fig. 1A). Basal pSTAT5 levels of $\mathrm{CD}^{+}{ }^{+}$Th cells were similar in AAV and HCs (given as MFI; $217 \pm 167$ vs. $154 \pm 72 ; \mathrm{P}=0.4)$.

Response of T cells to IL-2 is enhanced in AAV patients. IL-2 stimulation increased pSTAT5 levels of $\mathrm{CD}^{+} \mathrm{T}$ cells in $\mathrm{HCs}$ and AAV. The response to IL-2 was quantified as the ratio of pSTAT5 MFI, following stimulation with IL-2, divided by pSTAT5 MFI without a stimulus. The response to IL-2 stimulation was significantly higher in AAV patients compared with HC (2.8 \pm 1.4 vs. $1.8 \pm 1.1 ; \mathrm{P}=0.006$; Fig. $1 \mathrm{~B}$ and $\mathrm{C})$. Longitudinal measurements of AAV patients showed that the response to IL-2 was stable over time and persistently increased (Fig. 1D).

Constitutive pSTAT3 levels of $T$ cells are comparable in AAV and $\mathrm{HCs}$. Constitutive pSTAT3 levels of $\mathrm{CD}^{+} \mathrm{T}$ cells (given as MFI, $22 \pm 11$ vs. $26 \pm 11$; $\mathrm{P}=0.3$; Fig. $2 \mathrm{~A}$ ) and $\mathrm{CD}^{+}{ }^{+}$Th cells (given as MFI, $24 \pm 9$ vs. $27 \pm 7 ; \mathrm{P}=0.3$ ) were similar in $\mathrm{AAV}$ patients and HC. Stimulation of PBMC with IL-10 led to an in increase of pSTAT3 levels in $\mathrm{CD}^{+} \mathrm{T}$ cells of $\mathrm{HCs}$ and AAV patients. However, the response to IL-10 stimulation was significantly higher in AAV than in HCs $(2.9 \pm 1.2$ vs. $1.7 \pm 0.7$; $\mathrm{P}=0.001$; Fig. 2B and C).

pSTAT5/pSTAT3 expression in T cells and clinical implications. Notably, constitutive pSTAT3 expression of CD4 ${ }^{+}$ Th cells correlated with the relapse rate in patients with AAV ( $r=0.45 ; P=0.08$; Fig. 2D). By contrast, other clinical 
A

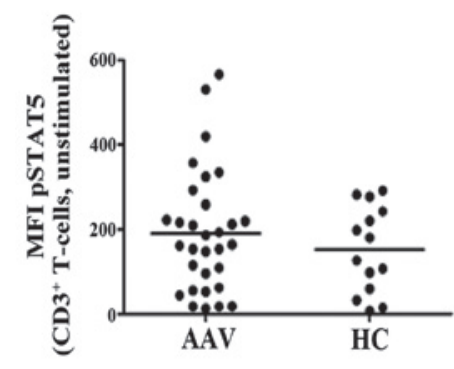

C

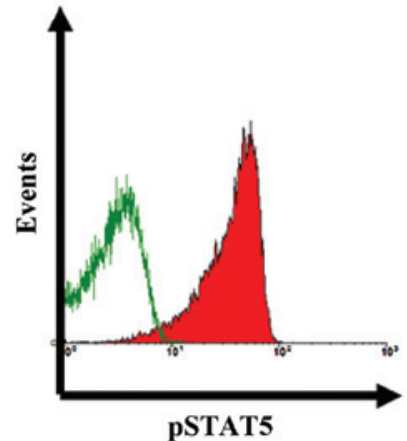

B

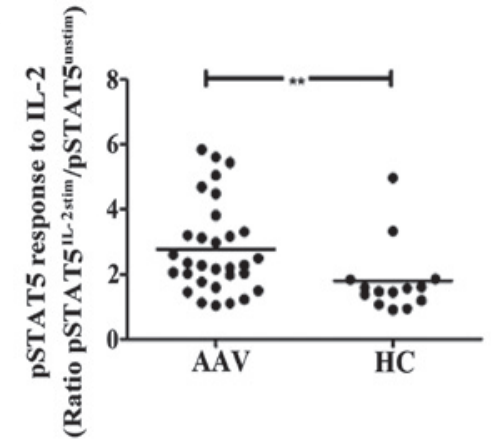

D

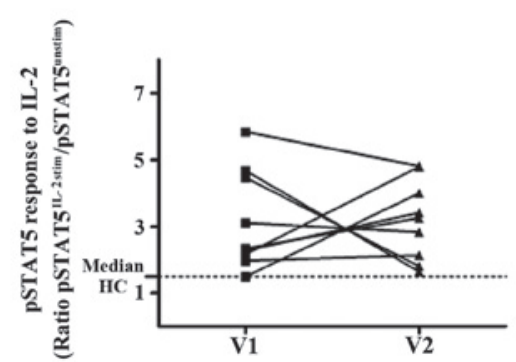

Figure 1. Constitutive and induced expression of phopshorylated STAT5 in CD3 ${ }^{+}$T cells. (A) The ex vivo constitutive levels of pSTAT5 are given as MFI. There was no significant difference between AAV patients and HCs. (B) Peripheral blood mononuclear cells were stimulated with $100 \mathrm{ng} / \mathrm{ml} \mathrm{IL}-2$ for $10 \mathrm{~min}$ and the MFI of pSTAT5 was determined. The response to IL-2 stimulation is given as a ratio of pSTAT5 MFI of stimulated T cells, divided by the pSTAT5 MFI of unstimulated T cells. The response to IL-2 was significantly elevated in AAV patients compared with HCs. (C) Representative raw data is shown as a histogram (green line, constitutive pSTAT5 expression of unstimulated CD3 ${ }^{+}$T cells; red shaded curve, pSTAT5 expression following stimulation with IL-2). The histogram was gated on $\mathrm{CD}^{+} \mathrm{T}$ cells. (D) The T-cell response to IL-2 stimulation was measured twice in $10 \mathrm{AAV}$ patients, (dotted line, median response of $\mathrm{CD}^{+} \mathrm{T}$ cells to IL-2 stimulation). The response to IL-2 is persistently elevated in patients with AAV ( ${ }^{* *} \mathrm{P}=0.006$, vs. HC group). MFI, mean fluorescence intensity; pSTAT5, phosphorylated signal transducer and activator of transcription 5; AAV, anti-neutrophil cytoplasmic antibody-associated vasculitis; HC, healthy control; IL-2, interleukin-2; CD3, cluster of differentiation 3.

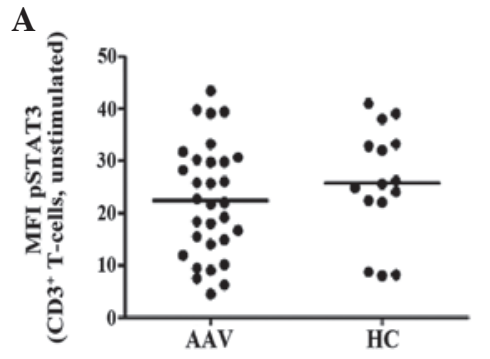

C

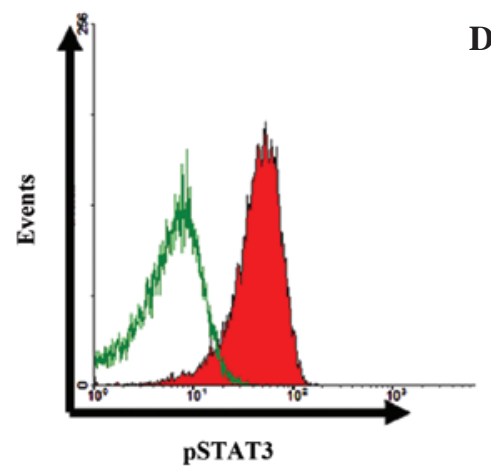

D
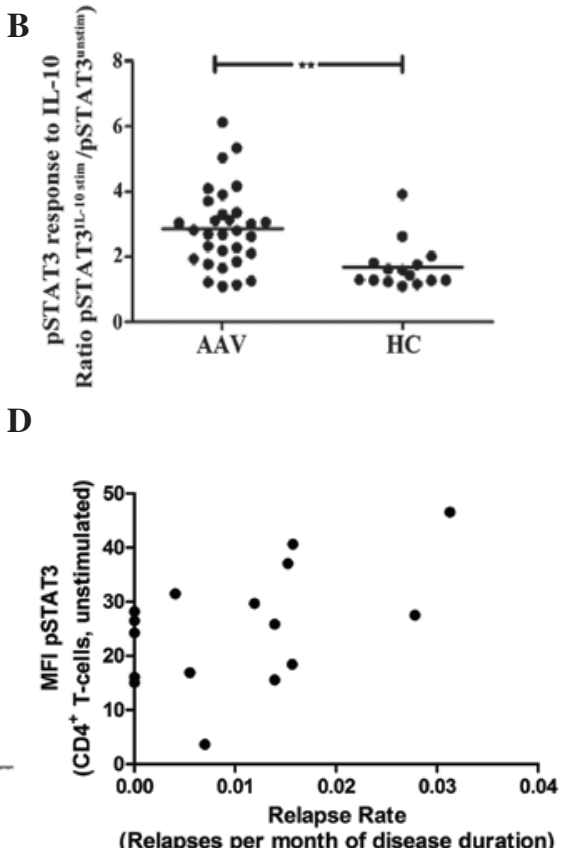

Figure 2. Constitutive and induced expression of phopshorylated STAT3 in $\mathrm{CD}^{+} \mathrm{T}$ cells. (A) The ex vivo constitutive levels of pSTAT3 are given as MFI. There was no significant difference between AAV patients and HCs. (B) Peripheral blood mononuclear cells were stimulated with $100 \mathrm{ng} / \mathrm{ml} \mathrm{IL-10} \mathrm{for} 10 \mathrm{~min}$ and the MFI of pSTAT3 was determined. The response to IL-10 stimulation is given as a ratio of pSTAT3 MFI of stimulated T cells, divided by the pSTAT3 MFI of unstimulated T cells. The response to IL-10 was significantly elevated in AAV patients compared with HCs. (C) Representative raw data is shown as a histogram (green line, constitutive pSTAT3 expression of unstimulated $\mathrm{CD} 3^{+} \mathrm{T}$ cells; red shaded curve, pSTAT3 expression following stimulation with IL-10). The histogram was gated on $\mathrm{CD}^{+} \mathrm{T}$ cells. (D) The ex vivo constitutive expression of pSTAT3 in $\mathrm{CD} 4^{+} \mathrm{T}$-helper cells correlated with the relapse rate in AAV patients $\left({ }^{* *} \mathrm{P}=0.001\right.$, vs. HC group). MFI, mean fluorescence intensity; pSTAT3, phosphorylated signal transducer and activator of transcription 3; AAV, anti-neutrophil cytoplasmic antibody-associated vasculitis; HC, healthy control; IL-10, interleukin-10; CD, cluster of differentiation. 
parameters, including renal function, disease extent, ANCA type and ANCA-positivity, were not associated with pSTAT3/5 expression of T cells (data not shown).

\section{Discussion}

The present study indicates that the constitutive expression of pSTAT5 and pSTAT3 within T cells is not altered in AAV. However, T cells from AAV patients responded with a greater increase of intracellular pSTAT5 and pSTAT3 to IL-2/10 stimulation. In addition, constitutive pSTAT3 expression correlated with the relapse propensity in vasculitis.

STAT5 and STAT3 are important regulators of T-cell immunity $(16,17)$. STAT5 is indispensable for the development of Treg cells, whilst STAT3 is essential for the differentiation of Th17 cells $(15,16,21)$. pSTAT5, the activated form of STAT5, directly interacts with the IL-17 gene locus and suppresses transcription (18). In addition, pSTAT5 enhances transcription of the Treg key transcription factor, FOXP3 $(17,18)$. AAV is characterized by a marked dysregulation of T-cell immunity $(2,7)$. Persistent expansion of Th17 cells and dysfunction of Treg cells have been confirmed in several previous studies $(1,2,7,14,27)$. Given the major role of pSTAT3/5 in regulation of T-cell immunity, the constitutive pSTAT3/5 expression of T cells in AAV patients was assessed in the present study. We hypothesized that a deficit in pSTAT5 and/or increased levels of pSTAT3 may cause an imbalance of Treg and Th17 cells (18). However, in contrast to this, ex vivo constitutive pSTAT3/5 expression levels of T cells were similar in $\mathrm{HC}$ and AAV. Constitutive pSTAT3 expression was associated with the relapse rate in $\mathrm{AAV}$, although this was not found to be statistically significant. This may indicate a pathophysiological role of the STAT3 pathway in AAV, but further studies are required to confirm this.

T-cell immunity and immune tolerance are critically affected by IL-2 (17). IL-2 signaling is mediated via the JAK/STAT5 pathway (17). Deficits in IL-2 signaling may cause aberrant Treg cell function and loss of immune tolerance (17). Therefore, the possible impairment of IL-2 signaling in AAV patients was analyzed. It was assessed whether IL-2 stimulation of $\mathrm{T}$ cells results in correct signal transduction and subsequent phosphorylation of STAT5. The response to IL-2 was much greater in T cells from AAV patients than from HCs. This may be due to an overexpression of the IL-2 receptor- $\alpha$ chain, CD25, on Th cells in AAV. Indeed, CD25 overexpression on $\mathrm{T}$ cells in AAV patients has been widely reported and likely causes the increased sensitivity to IL-2 stimulation $(8,9,11,27,28)$. Thus, IL-2 signaling appears intact and is unlikely to be the cause of Treg cell dysfunction in AAV, which had been reported previously $(14,27)$.

pSTAT3 is important in Th17 differentiation and is also a key signaling molecule in IL-10-mediated suppression of immune cells (20). IL-10 ligation to IL-10 receptors in T cells leads to activation of the JAK/STAT3 pathway and results in a rapid increase of intracellular pSTAT3 levels (20). Deficits in IL-10 signaling result in severe autoimmunity and loss of immune tolerance $(20,21)$. Therefore, the current study aimed to determine whether IL-10 stimulation of T cells of AAV patients results in correct signal transduction, as indicated by intracellular pSTAT3 levels. There was an increased response to IL-10 stimulation in AAV patients compared with HCs. However, the cause for this increased sensitivity is unclear. We hypothesize that the IL-10 receptor is overexpressed on T cells in AAV, as is the case with the IL-2 receptor- $\alpha$ chain. However, to the best of our knowledge, there have been no studies to date on IL-10 receptor expression on T cells in AAV. Alternatively, dephosphorylation of newly phosphorylated STAT3 by protein tyrosine phosphatases may be impaired, thereby inducing an increased IL-10 response $(15,16)$. However, the IL-10 signaling pathway in $\mathrm{T}$ cells appears to be undisturbed in AAV, as indicated by STAT3 phosphorylation.

In conclusion, constitutive expression of pSTAT5/3 is not altered in AAV. Signaling pathways for IL-2 and -10 , in which pSTAT5/3 are essential, are intact and functional in AAV. Thus, the T-cell abnormalities observed in AAV cannot be conclusively accounted for by alterations of STAT5- or STAT3-dependent pathways.

\section{References}

1. Wilde B, van Paassen P, Witzke O and Tervaert JW: New pathophysiological insights and treatment of ANCA-associated vasculitis. Kidney Int 79: 599-612, 2011.

2. Wilde B, Thewissen M, Damoiseaux J, van Paassen P, Witzke O and Tervaert JW: T cells in ANCA-associated vasculitis: what can we learn from lesional versus circulating T cells? Arthritis Res Ther 12: 204, 2010.

3. Brouwer E, Tervaert JW, Horst G, et al: Predominance of IgG1 and IgG4 subclasses of anti-neutrophil cytoplasmic autoantibodies (ANCA) in patients with Wegener's granulomatosis and clinically related disorders. Clin Exp Immunol 83: 379-386, 1991.

4. Wilde B, van Paassen P, Damoiseaux J, et al: Dendritic cells in renal biopsies of patients with ANCA-associated vasculitis. Nephrol Dial Transplant 24: 2151-2156, 2009.

5. Lamprecht P, Moosig F, Csernok E, et al: CD28 negative T cells are enriched in granulomatous lesions of the respiratory tract in Wegener's granulomatosis. Thorax 56: 751-757, 2001.

6. Lamprecht P, Csernok E and Gross WL: Effector memory T cells as driving force of granuloma formation and autoimmunity in Wegener's granulomatosis. J Intern Med 260: 187-191, 2006.

7. Wilde B, Thewissen M, Damoiseaux J, et al: Th17 expansion in granulomatosis with polyangiitis (Wegener's): the role of disease activity, immune regulation and therapy. Arthritis Res Ther 14: R227, 2012

8. Wilde B, Hua F, Dolff S, et al: Aberrant expression of the negative costimulator PD-1 on T cells in granulomatosis with polyangiitis. Rheumatology 51: 1188-1197, 2012.

9. Wilde B, Dolff S, Cai X, et al: $\mathrm{CD} 4{ }^{+} \mathrm{CD} 25^{+} \mathrm{T}$-cell populations expressing CD134 and GITR are associated with disease activity in patients with Wegener's granulomatosis. Nephrol Dial Transplant 24: 161-171, 2009.

10. Stegeman CA, Tervaert JW, Huitema MG and Kallenberg CG: Serum markers of T cell activation in relapses of Wegener's granulomatosis. Clin Exp Immunol 91: 415-420, 1993.

11. Popa ER, Stegeman CA, Bos NA, Kallenberg CG and Tervaert JW: Differential B- and T-cell activation in Wegener's granulomatosis. J Allergy Clin Immunol 103: 885-894, 1999.

12. Marinaki S, Kälsch AI, Grimminger P, et al: Persistent T-cell activation and clinical correlations in patients with ANCA-associated systemic vasculitis. Nephrol Dial Transplant 21: 1825-1832, 2006.

13. Abdulahad WH, Stegeman CA, van der Geld YM, Doornbos-van der Meer B, Limburg PC and Kallenberg CG: Functional defect of circulating regulatory $\mathrm{CD} 4^{+} \mathrm{T}$ cells in patients with Wegener's granulomatosis in remission. Arthritis Rheum 56: 2080-2091, 2007.

14. Free ME, Bunch DO, McGregor JA, et al: ANCA-associated vasculitis patients have defective Treg function exacerbated by presence of a suppression-resistant effector population. Arthritis Rheum 65: 1922-1933, 2013.

15. O'Shea JJ and Plenge R: JAK and STAT signaling molecules in immunoregulation and immune-mediated disease. Immunity 36 : $542-550,2012$. 
16. Stark GR and Darnell J Jr: The JAK-STAT pathway at twenty. Immunity 36: 503-514, 2012.

17. Liao W, Lin JX and Leonard WJ: Interleukin-2 at the crossroads of effector responses, tolerance, and immunotherapy. Immunity 38: 13-25, 2013.

18. Yang XP, Ghoreschi K, Steward-Tharp SM, et al: Opposing regulation of the locus encoding IL-17 through direct, reciprocal actions of STAT3 and STAT5. Nat Immunol 12: 247-254, 2011.

19. Lovato P, Brender C, Agnholt J, et al: Constitutive STAT3 activation in intestinal T cells from patients with Crohn's disease. J Biol Chem 278: 16777-16781, 2003.

20. Durant L, Watford WT, Ramos HL, et al: Diverse targets of the transcription factor STAT3 contribute to T cell pathogenicity and homeostasis. Immunity 32: 605-615, 2010.

21. Engelhardt KR, Shah N, Faizura-Yeop I, et al: Clinical outcome in IL-10- and IL-10 receptor-deficient patients with or without hematopoietic stem cell transplantation. J Allergy Clin Immunol 131: 825-830, 2013.

22. Hellmich B, Flossmann O, Gross WL, et al: EULAR recommendations for conducting clinical studies and/or clinical trials in systemic vasculitis: focus on anti-neutrophil cytoplasm antibody-associated vasculitis. Ann Rheum Dis 66: 605-617, 2007.
23. Leavitt RY, Fauci AS, Bloch DA, et al: The American College of Rheumatology 1990 criteria for the classification of Wegener's granulomatosis. Arthritis Rheum 33: 1101-1107, 1990.

24. Jennette JC, Falk RJ, Andrassy K, et al: Nomenclature of systemic vasculitides. Proposal of an international consensus conference. Arthritis Rheum 37: 187-192, 1994.

25. Jennette JC, Falk RJ, Bacon PA, et al: 2012 revised International Chapel Hill Consensus Conference Nomenclature of Vasculitides. Arthritis Rheum 65: 1-11, 2013.

26. Far DF, Peyron JF, Imbert V and Rossi B: Immunofluorescent quantification of tyrosine phosphorylation of cellular proteins in whole cells by flow cytometry. Cytometry 15: 327-334, 1994.

27. Morgan MD, Day CJ, Piper KP, et al: Patients with Wegener's granulomatosis demonstrate a relative deficiency and functional impairment of T-regulatory cells. Immunology 130: 64-73, 2010.

28. Marinaki S, Neumann I, Kälsch AI, et al: Abnormalities of CD4 $\mathrm{T}$ cell subpopulations in ANCA-associated vasculitis. Clin Exp Immunol 140: 181-191, 2005. 